\title{
Convergence Analysis of the African Buffalo Optimization Algorithm
}

\author{
Julius Beneoluchi Odili \\ FSKKP \\ odili_julest@yahoo.com
}

\author{
Mohd Nizam Mohmad Kahar \\ Faculty of Computer Systems and \\ Software Engineering \\ mnizam@ump.edu.my \\ Universiti Malaysia Pahang \\ Kuantan 26300, Malaysia.
}

\author{
A. Noraziah \\ IBM Centre of Excellence \\ noraziah@ump.edu.my
}

\begin{abstract}
This paper presents the convergence analysis of the newly-developed African Buffalo Optimization algorithm. African Buffalo Optimization is a simulation of the organizational skills of the African buffalos using two basic sounds: /waaa/ and /maaa/ as they transverse the African landscape in search of grazing pastures. The African Buffalo Optimization has proven to be quite successful since its development hence the need to examine its convergence behaviour. The analysis of the convergence of Natureinspired optimization algorithms is necessary to help researchers and practitioners understand the workings of the algorithms in the algorithms' attempts at solutions. After a number of evaluations, this study discovered that the convergence of African Buffalo Optimization is a function of the population size, communication topology, parameter-set, landscape topology and the objective function being optimized.
\end{abstract}

Keywords - African Buffalo Optimization, convergence analysis, communication topology, landscape topology, objective function.

\section{INTRODUCTION}

The African Buffalo Optimization (ABO) is a recently-developed metaheuristic algorithm. It was developed strictly following the lean metaheuristic design ideology being recently propagated by a number of researchers [1]. Basically, the ABO is a simulation of the movement of the African buffalos from place to place within the African forests and savannahs in search of lush pastures to satisfy their large appetites. In their movements, the African buffalos use the /maaa/ vocalizations to mobilize the animals to exploit a particular location and the opposing/waaa/ sounds to motivate each other to explore other areas since their present location is either unsafe or no more lucrative. Using these sounds, the buffalos are able to organize themselves out of starving locations into fruitful regions of the search space [2].

So far, the ABO has been successfully applied to solve different benchmark numerical test cases [3], asymmetric and symmetric Travelling Salesman's Problems as well as the tuning of Proportional, Integral and Derivative parameter-tuning of Automatic Voltage Regulators in Power generators [4].

In view of the huge success of the $\mathrm{ABO}$ in its areas of applications, our interest in this study is to investigate the Algorithms convergence behaviour. Earlier studies indicate that an algorithm's success is a function of number of parameters, effective tuning of such parameters, calculation of fitness of function, convergence behaviour, implementation strategies etc. $[5,6]$.

The convergence rate of a stochastic optimization algorithm is an indicator of its efficiency or otherwise. Algorithm convergence is an examination of the distribution of steps needed to reach the optimal solution.
The convergence analysis of the $\mathrm{ABO}$ is important since the novel algorithm is suitable not only for scientific investigations but also very useful in engineering applications. It is, therefore, necessary to provide the theoretical foundation of the novel algorithm in order to enable the research community appreciate its workings. This is the motivation for this study.

The rest of the paper is organized thus: section two discusses related work; section three examines the ABO algorithm and convergence analysis; section four highlights the findings on $\mathrm{ABO}$ convergence and presents the conclusion to the study. This is followed by the acknowledgement of support for the study

\section{RELATED WORK}

In recent years, a number of scientific investigations into the convergence behaviour of optimization algorithms has been carried out. Prominent among those investigation was the work by Liu et al [7] on the convergence of the Particle Swarm Optimization algorithm. In their study, Liu et al opines that PSO deploys 'the swarm intelligent model' in achieving good convergence. Swarm Intelligence Model postulates that the individual particles in a swarm relates with one another using iterated function system thus converging with a probability of individual particles being attracted towards the location of the overall best particle as the algorithm progresses from iteration to iteration. The movement of the other particles to the overall best in a particular iteration enables the PSO algorithm to converge at the global optimum. 
In another study on PSO convergence, Wei et al. opines that PSO achieves faster convergence using what they described as random global convergence theorem which is basically a simplification of the Swarm intelligence model described earlier [8]. Still in another study, Ren et al. used the Markov chain in analysing the global convergence characteristics of PSO [9]. Here, they established the relationship between the particles' velocity and positions in the swarm's movement from one location to the other as they move towards the global optima. In the study under reference, the researchers provided a theoretical relationship between the convergence time and convergence rate in addition to the relationship between the swarm size and convergence time.

The main issue in these approaches is the embedded assumption that that the swarm moves in one dimension.

Moreover, the PSO algorithm's convergence' time is calculated based on the particle interaction that depends on PSO's social-only component [10]. From the foregoing discussion, one prominent observations is that most studies on algorithm convergence are based on some rigid assumptions in order to simplify the theoretical foundations of the algorithm's convergence.

\section{THE ABO ALGORITHM}

To analyse the $\mathrm{ABO}$ convergence, it is necessary to take a look at the $\mathrm{ABO}$ algorithm as well as the workings of the algorithm. The ABO algorithm is presented in Figure 1 below.

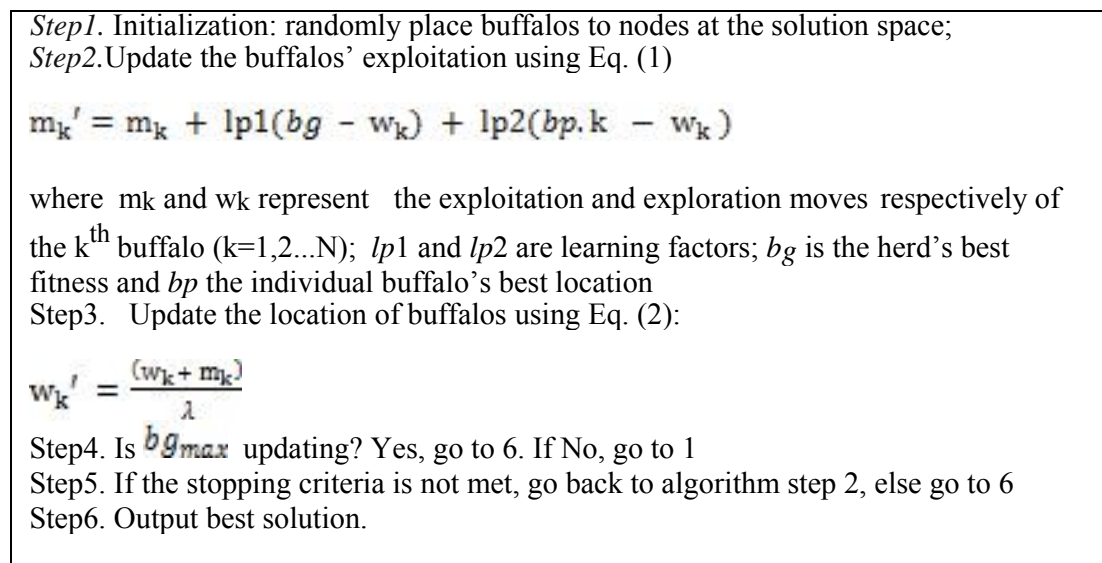

Figure 1: ABO algorithm

The ABO algorithm is basically a simulation of the three major characteristics of the African buffalos: regular communication, excellent memory capacity and collective intelligence borne out of regular consultations. The buffalos' communication is usually done through two vocalizations: the /waaa/ and the /maaa/. The calls (represented by in Fig. 1) are used to mobilize the herd to explore other grazing locations because the present location could be unsafe or lacks sufficient pasture. The other buffalo vocalization is the / call (represented by in Fig. 1) which summons the buffalos to stay on to exploit the present location since it has sufficient pasture and is safe. Mathematically, the democratic equation which simulates the cooperative interactions among the buffalos and determines their movement is:

$$
\mathrm{m}_{\mathrm{k}}{ }^{\prime}=\mathrm{m}_{\mathrm{k}}+\mathrm{lp} 1\left(b g-\mathrm{w}_{\mathrm{k}}\right)+\mathrm{lp} 2\left(b p \cdot \mathrm{k}-\mathrm{w}_{\mathrm{k}}\right)
$$

As can be seen, Eq. (1) has three main parts: the first being the memory part $\left(\mathrm{m}_{\mathrm{k}}{ }^{\prime}\right)$ which is an indication that the buffalos are aware that they have relocated from their locations, $m_{k}$ to the present one. This part of the Equation portrays the animals' extensive memory capacity which is an indispensable asset in their migrant lifestyle. The second part of Eq. (1) is a representation of the cooperative characteristics of the buffalos $\left(1 p 1\left(b q-w_{k}\right)\right.$. The buffalos are excellent communicators and so, in each iteration, are able to ascertain the location of the best buffalo which serves as a compass to the other buffalos in the search process. The last part of this equation $\operatorname{lp} 2\left(b p, \mathrm{k}-\mathrm{w}_{\mathrm{k}}\right.$ models the exceptional intelligence of the animals. They are able to tell their previous best fruitful location in comparison with the present position. The entire Eq. (1), therefore, highlights the buffalos' ability to harness the herd's collective intelligence in making informed decisions in their search for grazing lands [11].

It may be necessary to emphasize that the new exploitation location of the herd $\mathrm{mk}^{\prime}$ which will eventually determine the need for relocating to a new location wk' is a function of the previous exploitation location $w k$ in addition to the knowledge-based parameters $l p 1$ and $l p 2$ helping the buffalos in deciding the need for relocation after subtracting the present exploration location $\mathrm{wk}$ from their overall best $b g$ and personal best $b p . k$. Also note that $b g$ is derived by:

$$
b g=\mathrm{w} *(t)
$$


where $w *$ represents the best waaa (exploration) location and $t$ the iteration. Similarly, bp.k is obtained by:

$$
\text { bp. } \mathrm{k}=\mathrm{Wk}(t)
$$

Here $b p . \mathrm{k}$ is the best exploration location ever found by a particular buffalo $k$ and $t$ represents the iteration.

At this juncture, it is necessary to emphasize that the $\mathrm{ABO}$ algorithm subtracts the dimensional element $\mathrm{Wb}$ from the two maximum vectors ( $b g$ and $b p . \mathrm{k}$ ) and then multiplies the outcome by the learning parameters $\operatorname{lp} 1$, lp 2 which is usually between 0.3 and 0.7 . The output of these products is then used to determine the given fitness dimension ( $x$ or $v$ ) of the buffalos. At this point, Eq. 2 is then used to propel the buffalos to a new location:

$$
\mathrm{w}_{\mathrm{k}}^{\prime}=\frac{\left(w_{\mathrm{k}}+\mathrm{m}_{\mathrm{k}}\right)}{\lambda}
$$

Here $\mathrm{Wk}_{\mathrm{k}}$ 'represents the movement to a new location, $\mathrm{W}_{\mathrm{k}}$ is the present exploration values while $\mathrm{m}_{\mathrm{k}}$ is the present exploitation values; $\lambda$ represents exploitation driver is a random between 0 and 1. The Algorithm flowchart is presented in Figure 2.

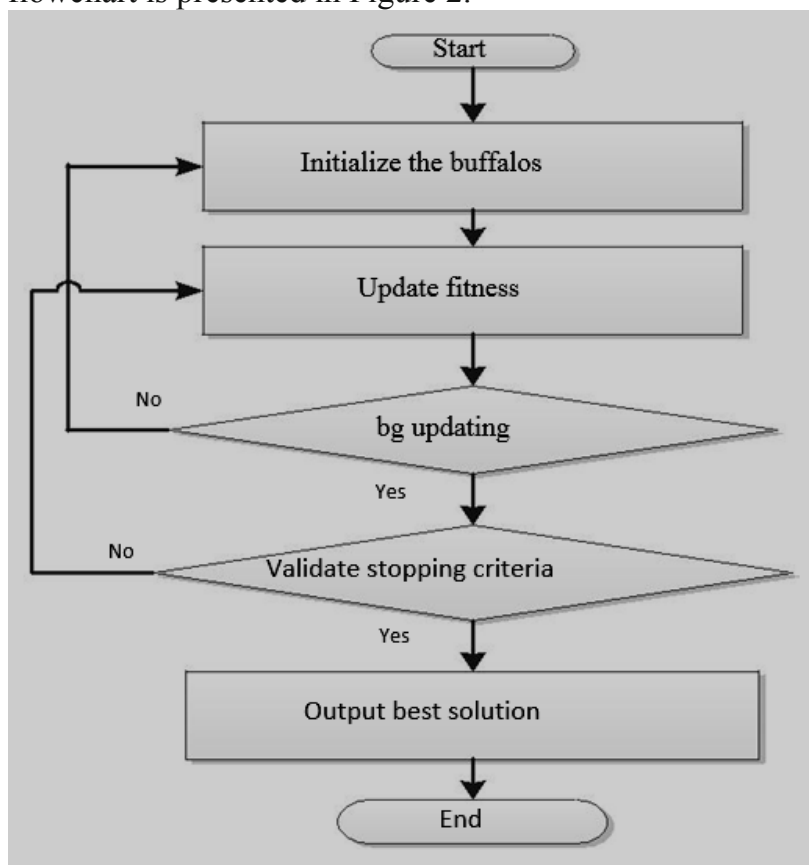

Figure 2: The flowchart of ABO

\section{A. The Working of the $A B O$.}

As can be seen from Fig. 1, the ABO algorithm starts by randomly initializing the population of buffalos. This is done by assigning random locations to each buffalo within the search space. After this, it updates each buffalo's exploitation and exploration fitness in relation to the target goal. This way it ascertains the herd's best animal ( and each buffalos personal best ( ). Please note that in each step, each animal keeps a memory of its own coordinates. If the present fitness is better than the individual buffalo's maximum fitness ( ), the algorithm saves that location vector for the particular buffalo. If the fitness is better than the herd's maximum, it saves it as the herd's global best ( )..

After this, the algorithm updates the location of the buffalos using Eq.2. Moreover, it confirms the improvement or otherwise of the best buffalo ( ). If there is no improvement in the status of the best buffalo in a number of iterations, the algorithm re-initializes the entire buffalo herd. If the best buffalo is improving its locations, the algorithm checks to see if the stopping criteria has been reached. If the best buffalo fitness ( meets our exit criteria, it terminates the run and provides the location vector as the solution to the given problem. The stopping criteria could be a specified number of iterations without improvement of the best buffalo, a specified number of iterations etc. Some of the advantages of this new algorithm are its ease of implementation, ability to search both locally and globally simultaneously, the algorithm's flexibility, the use of relatively few parameters and fast convergence rate.

\section{B. ABO Convergence Analysis.}

There exists a relationship between the exploitation driver of the population and the algorithm convergence. Equation 3 below defines the population's fitness in terms of since which represents the exploitation driver is a major determinant of the buffalos' movement. A higher value of means more exploitation, less exploration and vice-versa:

$$
\lambda=\quad \frac{1}{N} \sum_{l=1}^{N}\left(\frac{w_{k}-w_{\text {avg }}}{w j}\right)
$$

Here $\mathrm{N}$ stands for the population of buffalos; wk is the /waaa/ (exploration) fitness of buffalo $\mathrm{k}, \mathrm{k}=1,2,3$,..n ; Wavg, the present average (exploration) fitness of the herd; while $\mathrm{Wj}_{\mathrm{j}}$ is the normalized calibration factor of the exploitation driver. The value of $\mathrm{w}$ is obtainable from:

$$
w=\max \left(\left(\left|w_{k}-w_{\text {avg }}\right| k, \max \right)\right), k \in[1, \mathrm{~N}]
$$

The way is defined is an indication that being the exploitation driver it presents the convergence degree of all the buffalos in the herd. It follows, therefore, that a bigger $\lambda$ presents a better convergence. Otherwise, the buffalos are still in the random searching mode. Another way to describe the buffalos' convergence is: 


$$
\lim k(t)=j
$$

In this Equation, $k(t)$ represents the /waaa/values of the buffalo $k$ at iteration $t$ and $i$ the herd's location in the searching space. This shows that the buffalos will settle at some fixed point $i$ in the search space. Mathematically, this can be represented as:

$$
\lim _{n \rightarrow \infty} k(t)=\theta * b_{p}+(1-\theta) * b_{g}
$$

Here, $\theta=l p 1 /(l p 1+l p 2), \quad l p 1$ and $l p 2$ and are learning parameters in equation (1), and $b_{n}$ is the individual best location while $b_{a}$ is the global best of the herd.

In summary, therefore, $\mathrm{ABO}$ arrives at convergence in a local or global extremum: the entire buffalos aggregating in a location, with the variance of the population's fitness being zero. Practical application of the $\mathrm{ABO}$ convergence in parameter-tuning is found in the Appendix. The best parameter tuple is in green colour while the next best is in red.

\section{Communication Issues in ABO Convergence.}

Different optimization algorithms employ diverse strategies in searching for solutions to optimization problems. For example, the Ant Colony Optimization (ACO) uses the path construction method in its search while the Particle Swarm Optimization (PSO), Artificial Bee Colony $(\mathrm{ABC})$ and the $\mathrm{ABO}$ use the path improvement mechanism.

However, while the ACO's best information propagation method is the ring topology [12], the PSO's best communication topology is the Von Neumann architecture [13], while the ABO employs the star topology which connects all the buffalos together since the entire herd works as a unit in search of solutions for its information propagation [14]. A diagrammatic representation of the different communication topologies are presented in Figure 3 below.

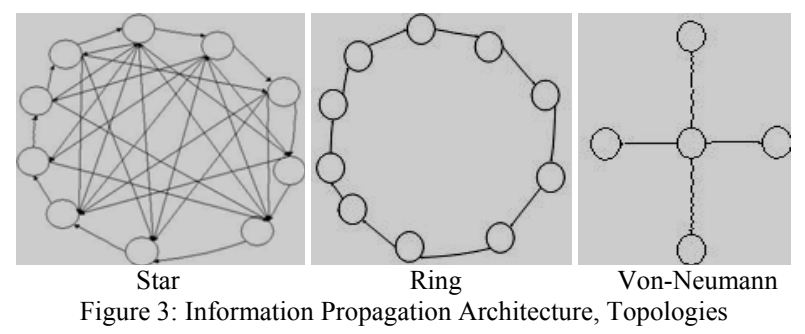

\section{FINDINGS AND CONCLUSIONS}

Convergence basically is the trade-off between exploration and exploitation. In order to obtain a good convergence of the ABO, it is necessary to select the appropriate parameters. The algorithm's convergence is a function of the population of the buffalos engaged in the search, the communication topology, size of the search space, the function being optimized in terms of the number of local optima and their distance to the global optimum, the position of the global optimum etc. This is in agreement with previous studies $[15,16]$

Experimental procedures indicate that larger population sizes demand that the algorithm embarks on several evaluations per iteration and this impedes on the convergence speed. Similarly, the communication topology adopted whether the star, ring or Von Neumann influences the algorithm's convergence. The star topology, for instance, ensures fast communication and consequently fast convergence; the ring or Von Neumann are slower communication models and, therefore, slower convergence.

Another major factor in $\mathrm{ABO}$ convergence is the nature of the problem being solved. The function being optimized could be such that have several local optima or otherwise. It is easier to solve a problem with fewer local optima starched together than a function that has several local optima spread all over the search landscape. Closely related to this is the position of the local optima in relation to the global optimum. The distance of the global optimum in relation to the local optima is a major consideration affecting the rate of ABO's convergence. The algorithm is most likely to converge faster in a situation where the global optimum is close to the local optima. Still on the issue of the global optimum location is its position whether in the centre of the search space or nearer to the borders. Experimental investigation shows that global optimum at the centre of the search space is easier to locate, thus ensuring faster convergence than those near the borders. This finding is consistent with earlier results $[17,18]$.

Moreover, it is pertinent to note that the size of the search space is a major consideration in $\mathrm{ABO}$ convergence processes: the larger the search space, the more time and computer resources taken by the buffalos in their search before convergence than in smaller search spaces. Also noteworthy is the search spaces with deceptive surfaces. Very smooth search spaces as in Rosenbrock test function poses a great challenge to the $\mathrm{ABO}$ as it gives insufficient information to the algorithm in its search effort.

In the light of the above discussion, it is advisable to start with a particular parameter set and observe the convergence outcome. In a situation where different results are obtained in most runs, this is an indication that the algorithm convergence is rather too fast. In such a situation, the population of buffalos should be increased or the communication topology be loosened. That is to say, that if one is using the star topology, there could be need to deploy the Von Neumann communication strategy or even the ring topology.

Conversely, if the runs produce the same outcomes 
consistently, yet no improvement is made during a good proportion of the run, especially, when the outcome is not the expected global optimum then it is a case of sluggish convergence. However, if the outcomes of most runs are consistent with noticeable improvements per iterations, then it is a case of proper convergence speed. In all of these cases, it can be deduced that the lambda helps the $\mathrm{ABO}$ to determine the steps of the buffalos in their quest for solutions.

In closing, it is obvious from the fore-going analysis that the effectiveness and efficiency of the ABO is a function of its convergence behaviour. Also, the convergence characteristics of the ABO algorithm is a function of the population size being deployed for each problem, the communication topology employed to solve a problem, the parameter-set being used in the search, the landscape topology of the problem being solved and the objective function being optimized.

\section{ACKNOWLEDGMENT}

The authors appreciate the support of the Faculty of Computer Systems and Software Engineering, Universiti Malaysia Pahang, Kuantan 26300, Malaysia under Grant GRS 1403118. Our gratitude to Ministry of Higher Education, Malaysia for additional funding under FRGS Grant RDU140101

\section{REFERENCES}

[1] J. B. Odili, M. N. M. Kahar, and S. Anwar, "African Buffalo Optimization: A Swarm-Intelligence Technique," Procedia Computer Science, 2015.

[2] J. B. Odili and M. N. M. Kahar, "African Buffalo Optimization (ABO): a New Meta-Heuristic Algorithm," Journal of Advanced \& Applied Sciences, vol. 03, pp. 101-106, April 20152015.

[3] J. B. Odili and M. N. M. Kahar, "Numerical Function Optimization Solutions Using the African Buffalo Optimization Algorithm (ABO)," British Journal of Mathematics \& Computer Science, vol. 10, pp. 1-12, 2015.

[4] J. B. Odili and M. N. Mohmad Kahar, "Solving the Traveling Salesman's Problem Using the African Buffalo Optimization," Computational Intelligence and Neuroscience, vol. 501, p.
929547, 2015.

[5] J. M. Huntley and H. O. Saldner, "Shape measurement by temporal phase unwrapping: comparison of unwrapping algorithms," Measurement Science and Technology, vol. 8, p. 986, 1997.

[6] X.-S. Yang, "Nature-inspired metaheuristic algorithms: success and new challenges," arXiv preprint arXiv:1211.6658, 2012.

[7] H. Liu, A. Abraham, and V. Snásel, "Convergence Analysis of Swarm Algorithm," in NaBIC, 2009, pp. 1714-1719.

[8] F. Wei, S. Jun, X. Zhen-Ping, and X. Wen-Bo, "Convergence analysis of quantum-behaved particle swarm optimization algorithm and study on its control parameter," 2010.

[9] Z.-H. Ren, J. Wang, and Y.-L. Gao, "The global convergence analysis of particle swarm optimization algorithm based on Markov chain," Control Theory \& Applications, vol. 28, pp. 462466, 2011.

[10] C.-H. Chen and Y.-p. Chen, "Convergence time analysis of particle swarm optimization based on particle interaction," Advances in Artificial Intelligence, vol. 2011, p. 7, 2011.

[11] J. B. Odili, M. N. M. Kahar, S. Anwar, and M. A. K. Azrag, "A comparative study of African Buffalo Optimization and Randomized Insertion Algorithm for asymmetric Travelling Salesman's Problem," in Software Engineering and Computer Systems (ICSECS), 2015 4th International Conference on, Kuantan, Malaysia, 2015, pp. 90-95.

[12] A. M. Mora, P. García-Sánchez, J. J. Merelo, and P. A. Castillo, "Migration study on a pareto-based island model for MOACOs," in Proceedings of the 15th annual conference on Genetic and evolutionary computation, 2013, pp. 57-64.

[13] J. Kennedy and R. Mendes, "Population structure and particle swarm performance," 2002.

[14] T. Kurihara and K. Jin'no, "Analysis of convergence property of PSO and its application to nonlinear blind source separation," in Evolutionary Computation (CEC), 2013 IEEE Congress on, 2013, pp. 976-981.

[15] Y.-x. Yuan, "Problems on convergence of unconstrained optimization algorithms," Numerical Linear Algebra and Optimization, (Science Press, Beijing, New York), pp. 95-107, 1999.

[16] D. Su, J. Dong, and Z. ZHENG, "A stochastic algorithm for function minimization," Optimization online, 2008.

[17] I. C. Trelea, "The particle swarm optimization algorithm: convergence analysis and parameter selection," Information processing letters, vol. 85, pp. 317-325, 2003.

[18] T. Kurihara and K. Jin'no, "Analysis of convergence property of PSO and its application to nonlinear blind source separation," in 2013 IEEE Congress on Evolutionary Computation, 2013, pp. 976-981. 
APPENDIX

\begin{tabular}{|c|c|c|c|c|}
\hline$\lambda$ & lp1 & lp2 & Best result & Mean score \\
\hline & & 0.3 & -10.3923 & -10.3144 \\
\hline & & 0.4 & -10.3923 & -10.3124 \\
\hline & & 0.5 & -10.3922 & -10.3380 \\
\hline & & 0.6 & -10.3911 & -10.2235 \\
\hline & & 0.7 & -10.3923 & -10.3448 \\
\hline & & 0.8 & -10.3923 & -10.3547 \\
\hline & & 0.9 & -10.3923 & -10.1674 \\
\hline \multirow[t]{27}{*}{1.8} & 0.7 & 0.1 & -10.3917 & -10.3562 \\
\hline & & 0.2 & -10.3905 & -10.2924 \\
\hline & & 0.3 & -10.3923 & -10.3779 \\
\hline & & 0.4 & 10.3912 & -10.1720 \\
\hline & & 0.5 & -10.3923 & -10.3576 \\
\hline & & 0.6 & -10.3923 & -10.3012 \\
\hline & & 0.7 & -10.3923 & -10.1275 \\
\hline & & 0.8 & -10.3923 & -10.3422 \\
\hline & & 0.9 & -10.3923 & 10.3825 \\
\hline & 0.8 & 0.1 & -10.3912 & -10.2523 \\
\hline & & 0.2 & -10.3923 & -10.3553 \\
\hline & & 0.3 & -10.3923 & -10.3470 \\
\hline & & 0.4 & -10.3923 & -10.3757 \\
\hline & & 0.5 & -10.3922 & -10.1728 \\
\hline & & 0.6 & -10.3921 & -10.1681 \\
\hline & & 0.7 & -10.3916 & -10.3168 \\
\hline & & 0.8 & -10.3923 & -10.2993 \\
\hline & & 0.9 & -10.3923 & -10.3814 \\
\hline & 0.9 & 0.1 & -10.3923 & -10.2956 \\
\hline & & 0.2 & -10.3922 & -10.3205 \\
\hline & & 0.3 & -10.3923 & -10.3503 \\
\hline & & 0.4 & -10.3923 & -10.2918 \\
\hline & & 0.5 & -10.3923 & -10.2893 \\
\hline & & 0.6 & -10.3923 & -10.3609 \\
\hline & & 0.7 & -10.3923 & -10.3730 \\
\hline & & 0.8 & -10.3923 & -10.3852 \\
\hline & & 0.9 & -10.3923 & -10.3553 \\
\hline \multirow[t]{10}{*}{1.6} & 0.1 & 0.1 & -10.3912 & -10.2627 \\
\hline & & 0.2 & -10.3923 & -10.2407 \\
\hline & & 0.3 & -10.3918 & -10.1043 \\
\hline & & 0.4 & -10.3921 & -10.1112 \\
\hline & & 0.5 & -10.3923 & -9.7980 \\
\hline & & 0.6 & -10.3907 & -10.1446 \\
\hline & & 0.7 & -10.3921 & -10.1703 \\
\hline & & 0.8 & -10.3921 & -10.0376 \\
\hline & & 0.9 & -10.3923 & -10.1564 \\
\hline & 0.2 & 0.1 & -10.3923 & -10.3180 \\
\hline
\end{tabular}

